\title{
Signatures of star streams in the phase space distribution of nearby halo stars
}

\author{
C. Dettbarn ${ }^{1}$, B. Fuchs ${ }^{1}$, C. Flynn ${ }^{2,3}$, and M. Williams ${ }^{3}$ \\ 1 Astronomisches Rechen-Institut am Zentrum für Astronomie der Universität Heidelberg, Mönchhofstr. 12-14, 69120 Heidelberg, \\ Germany \\ e-mail: dettbarn@ari.uni-heidelberg.de \\ 2 Tuorla Observatory, University of Turku, Väisäläntie 20, 21500 Piikkiö, Finland \\ 3 Mt. Stromlo Observatory, Australian National University, Cotter Rd., Weston Creek ACT 2611, Australia \\ Received 13 March 2007 / Accepted 21 August 2007
}

ABSTRACT

\begin{abstract}
We have analyzed the phase space distribution of a sample of about 900 non-kinematically selected low metallicity stars in the solar vicinity. The stars primarily represent the thick disk and halo populations of the Milky Way. We aim to identify overdensely populated regions in phase space, which we interpret as signatures of star streams passing close to the Sun. The search was conducted in a space constructed from the angular momenta and eccentricities of the stellar orbits. Besides recovering all well known star streams in the thick disk, we isolated four statistically significant phase space overdensities amongst halo stars. One of them is associated with a previously known halo star stream, but three of them are novel features, which we propose be also considered as genuine halo streams.
\end{abstract}

Key words. Galaxy: halo - Galaxy: kinematics and dynamics

\section{Introduction}

The recent large scale surveys of the stellar population of the Milky Way have revealed the presence of long coherent stellar streams. Most spectacular are the Sagittarius stream, which wraps around the entire Galaxy nearly perpendicular to the midplane, (Ibata et al. 2001; Martínez-Delgado et al. 2001; Majewski et al. 2003; Belokurov et al. 2006), and at low Galactic latitudes the Monoceros stream (Yanny et al. 2003; Ibata et al. 2003). These are interpreted as tidal debris associated with disrupted satellite galaxies, which were accreted by the Milky Way. Numerical simulations of such merger events have shown that such debris streams survive as coherent structures over gigayears so that they are witnesses of the formation history of the Galaxy (Helmi 2004; Law et al. 2005; Peñarrubia et al. 2005).

Stellar streams have also been discovered as overdensities in the phase space distribution of stars in the solar neighbourhood. Helmi et al. (1999) detected signs for a star stream in the halo population passing close to the Sun which was later confirmed by Chiba \& Beers (2000). Prominent streams or more correctly 'moving groups' in the disk population of stars in the vicinity of the Sun are the Hercules, Pleiades-Hyades, and Sirius streams (Eggen 1996; Dehnen 1998). These moving groups are very probably not related to tidal debris streams, but originate dynamically within the disk itself. Dehnen (2000) has demonstrated that stars in the Hercules group are presumably in outer Lindblad resonance with the inner bar of the Milky Way which leads to focussing of stellar orbits into certain parts of phase space. Quillen \& Minchev (2005) have suggested that the other moving groups are due to resonances with spiral density waves. All these features can be traced both in the thin and the thick disk populations. The Arcturus stream (Eggen 1996), on the other hand, is attributed to the thick disk only and is probably of extragalactic origin (Navarro et al. 2004). Arifyanto \& Fuchs (2006) have analyzed the kinematics of nearby subdwarfs and identified another stream in the thick disk which resembles closely the Arcturus stream. The same feature has been detected independently by Helmi et al. (2006) in the phase space distribution of nearby $\mathrm{F}$ and $\mathrm{G}$ stars. The nature of this stream is at present unclear. Helmi et al. (2006) argue in favour of an extragalactic origin, because they find that its phase space structure resembles that of numerically simulated debris streams of disrupted satellite galaxies. On the other hand, it could as well owe its existence to similar dynamical effects which caused the Hercules group. If the members of the Hercules group are at outer Lindblad resonance with the Galactic bar (Dehnen 2000), the members of the new group would be at the outer 4:1 ultraharmonic resonance with the bar (Fuchs, unpublished). These questions can only be settled, if the elemental abundance pattern of the members of the streams are known. They would indicate that either the stars belong to the field population of stars in the Milky Way or to the population of a disintegrated dwarf galaxy with different abundances (Williams \& Freeman, in preparation).

In this study we investigate the phase space distribution of halo stars in the solar neighbourhood, searching for signatures of streams in the Galactic halo.

\section{Search strategy for star streams in the halo population}

Our analysis is based on the sample of non-kinematically selected low metallicity stars of Beers et al. (2000). We have drawn from that catalogue the space velocity data of all stars with metallicties $[\mathrm{Fe} / \mathrm{H}]<-1$, which leads to a sample of 897 stars. To the heliocentric space velocity vector $(U, V, W)$ of each star, with $U$ pointing towards the Galactic center, $V$ into the direction of Galactic rotation, and $W$ towards the North Galactic Pole, respectively, the velocity vector of the motion of the Sun relative to the local standard of rest $\left(U_{\odot}, V_{\odot}, W_{\odot}\right)=(10.0,5.3,7.2) \mathrm{km} \mathrm{s}^{-1}$ was added (Dehnen \& Binney 1998). Next, the velocities are 

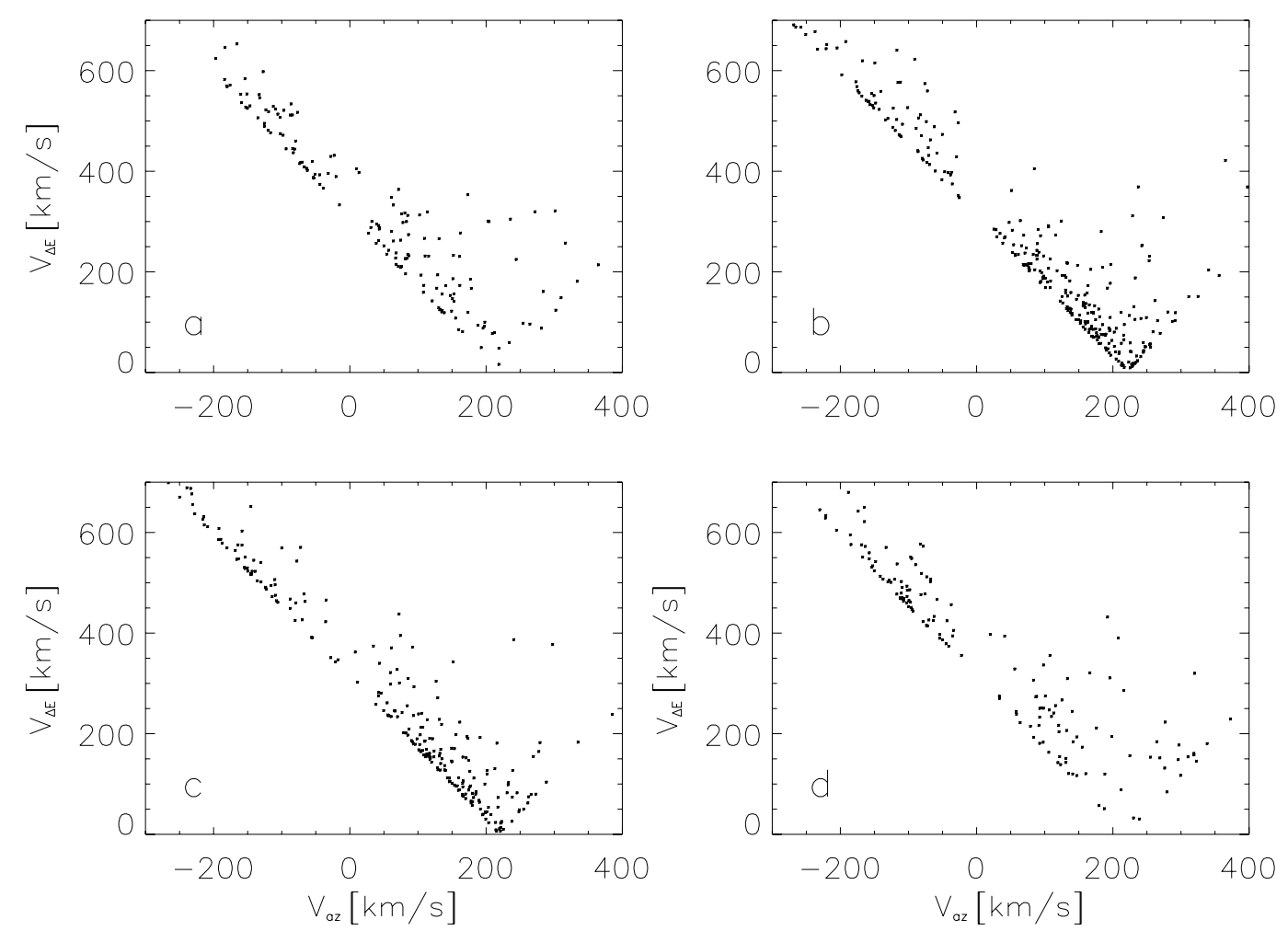

Fig. 1. Slices through the phase space distribution of halo stars in the solar neighbourhood. The panels a), b), c), and d) show the velocities of stars on orbital planes inclined relative to the direction towards the North Galactic Pole by angles of $v=0^{\circ}-45^{\circ}, 45^{\circ}-90^{\circ}, 90^{\circ}-135^{\circ}$, and $135^{\circ}-180^{\circ}$, respectively (top left to bottom right). $V_{\mathrm{az}}$ denotes the tangential velocity of the stars and $V_{\mathrm{AE}}$ is a measure of the eccentricities of the stellar orbits (cf. Sect. 2).

transformed from the reference frame of the local standard of rest to an inertial frame by adding to the $V$ velocity components the circular velocity of the local standard of rest around the Galactic center, for which we adopt the value of $V_{\mathrm{LSR}}=$ $220 \mathrm{~km} \mathrm{~s}^{-1}$. In the following we assume a spherical Galactic gravitational potential and neglect the flattening of the disk potential and any asphericity of the dark halo potential. Chiba \& Beers (2000) have modelled the Galactic gravitational potential with aspherical Stäckel potentials. They showed that the distribution of halo stars in the phase space spanned by the isolating integrals of motion of the stellar orbits in such a potential can be closely mapped into the corresponding phase space constructed assuming a spherical potential, which justifies our procedure.

In a spherical potential a star moves in a constant orbital plane. Most of the stars which we have extracted from the Beers et al. (2000) sample have distances less than $1 \mathrm{kpc}$ from the Sun, and for the purpose of calculating orbital parameters may be assumed to be at the Sun. The azimuthal velocity of a star is obviously given by $V_{\mathrm{az}}=\sqrt{\left(V+V_{\mathrm{LSR}}\right)^{2}+W^{2}}$ and the angular momentum is then $L=R_{\odot} \cdot V_{\text {az }}$, where $R_{\odot}$ denotes the galactocentric distance of the Sun for which we assume $R_{\odot}=8 \mathrm{kpc}$. The inclination angle of the orbital plane relative to the direction towards the North Galactic Pole is given by $v=\arctan \left(\left(V+V_{\mathrm{LSR}}\right) / W\right)$. We restrict in our analysis the inclination angles to the range $0^{\circ} \leq v<180^{\circ}$. Stars in an orbital plane with an inclination angle larger than $180^{\circ}$ are treated as retrograde stars in the orbital plane with an inclination angle $v-180^{\circ}$. The aim of our study is to identify star streams among the halo stars. Thus we are searching for groups of stars on essentially the same orbits which pass at present through the solar neigbourhood. For this purpose we have generalized the search technique of Arifyanto $\&$ Fuchs (2006) and have grouped the stars in our sample according to the inclination angles of their orbital planes. In each $v$-slice we have then searched for overdensities in a space spanned by angular momentum, which defines the mean guiding center radius of a stellar orbit, and eccentricity, which describes the radial excursion of the orbit from its mean guiding center radius. Since all stars are at present close to the Sun, they all have the same phase along their orbits. Using the theory of Galactic orbits by Dekker (1976) Arifyanto \& Fuchs (2006) have shown that the search can be conducted in practice in the space of $V_{\mathrm{az}}$ and $V_{\Delta \mathrm{E}}=\sqrt{U^{2}+2\left(V_{\mathrm{az}}-V_{\mathrm{LSR}}\right)^{2}}$. $V_{\mathrm{az}}$ is directly related to the angular momentum and $V_{\Delta \mathrm{E}}$, defined by the difference between the actual orbital energy and the orbital energy of a star on the circular guiding center orbit, is a measure of the eccentricity of the orbit, $e=V_{\Delta \mathrm{E}} / \sqrt{2} V_{\mathrm{LSR}}$. The latter assumes a flat Galactic rotation curve. $V_{\Delta \mathrm{E}}$ is also related to the radial action integral of the orbit and should be quite robust, even if the Galactic potential underwent changes in the past. In order to enhance the overdensities of stars in phase space we have performed a three-dimensional wavelet transform with a Mexican hat kernel $\mathcal{K}\left(\boldsymbol{x}-\boldsymbol{x}^{\prime}\right)=\left(3-\left(\boldsymbol{x}-\boldsymbol{x}^{\prime}\right)^{2} / a^{2}\right) \exp -\left(\left(\boldsymbol{x}-\boldsymbol{x}^{\prime}\right)^{2} / 2 a^{2}\right)$ (cf. Skuljan et al. 1999) of the $V_{\mathrm{az}}-V_{\Delta \mathrm{E}}-v$ data cube. After some experimentation we adopted a wavelet length scale of $a=20 \mathrm{~km} \mathrm{~s}^{-1}$ which leads to the clearest results. The wavelet transform conserves the total size of the occupied phase space, because the volume integral over $\mathcal{K}$ is equal to zero.

\section{Results and discussion}

\subsection{Star streams in the halo population}

In Fig. 1 we show scatter plots of slices through the threedimensional phase space distribution of the stars in our sample. 

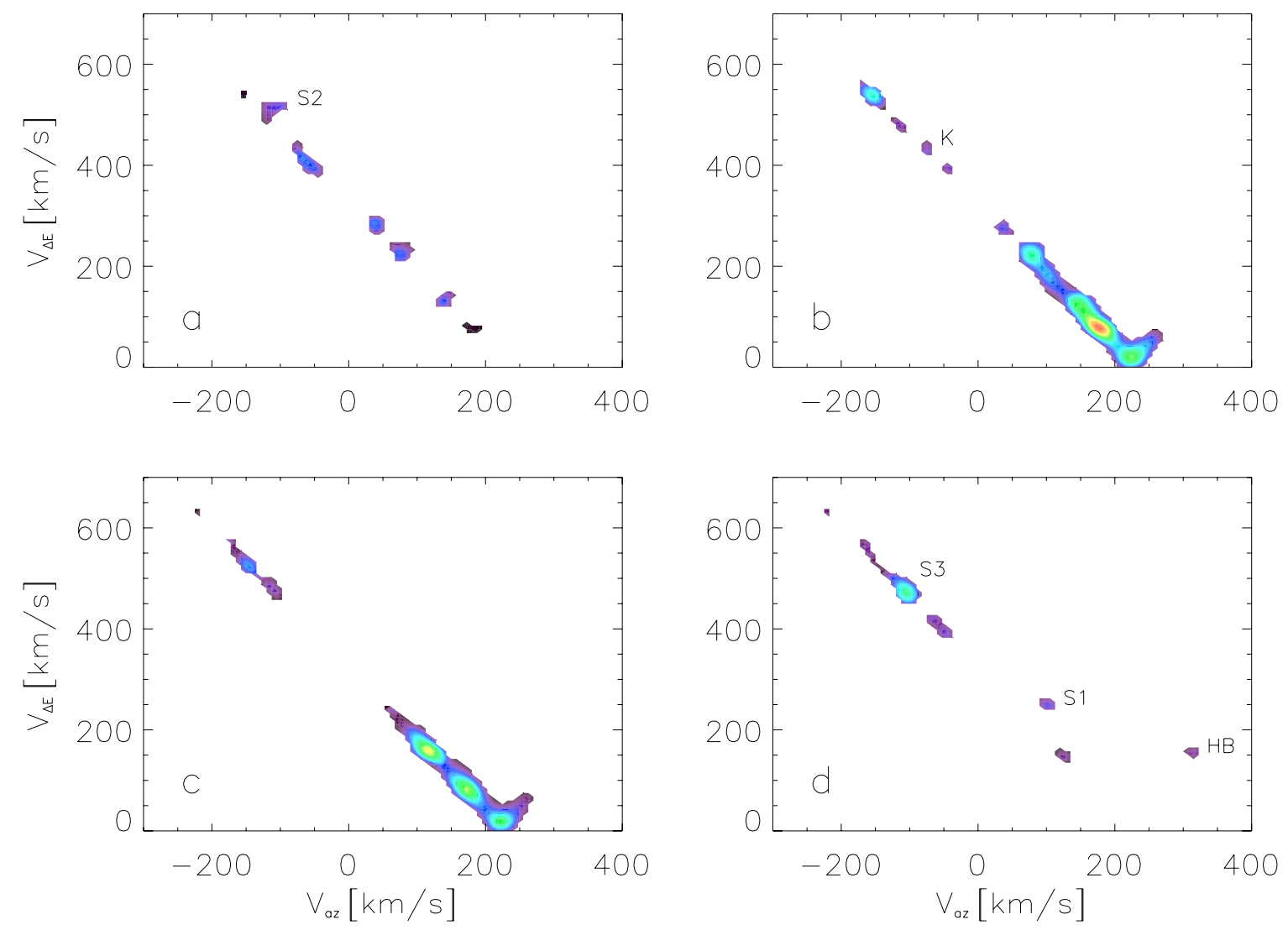

Fig. 2. Slices through the wavelet transform of the phase space distribution of halo stars in the solar neighbourhood constructed analogous to the slices in Fig. 1. The phase space densities are colour coded at $10 \%$ levels according to a linear colour table ranging from red, over yellow, green, blue to dark violet. The labels indicate halo star streams.

Each slice is $45^{\circ}$ wide, and covers $0^{\circ}$ to $45^{\circ}, 45^{\circ}$ to $90^{\circ}, 90^{\circ}$ to $135^{\circ}$, and $135^{\circ}$ to $180^{\circ}$, respectively. Corresponding slices through the three-dimensional wavelet transforms of the data, which enhance and delineate the overdensities in a quantitative way, are shown in Fig. 2. A different projection of the threedimensional data cube onto inclination angle $v$ versus azimuthal velocity $V_{\mathrm{az}}$ is shown in Fig. 3. Since the $\mathrm{V}$-shaped features in the $V_{\mathrm{az}}-V_{\Delta \mathrm{E}}$ planes are rather narrow, we have integrated in Fig. 3 all phase space densities over $V_{\Delta \mathrm{E}}$. A three-dimensional rendering of the data cube is shown in Fig. 4. As can be seen from Figs. 2-4 there is a lot of fine structure in the phase space distribution of the stars. In the most fully populated part of phase space the inclination angles of the orbital planes are about 90 degrees. These stars have disk-like kinematics and most of them are even at these comparatively low metallicities members of the thick disk of the Galaxy. The diagrams in the upper right and lower left panels of Fig. 2 show clearly the Hercules stream at $V_{\mathrm{az}}=170 \mathrm{~km} \mathrm{~s}^{-1}$, the stream found by Arifyanto \& Fuchs (2006) and Helmi et al. (2006) at $V_{\mathrm{az}}=120 \mathrm{~km} \mathrm{~s}^{-1}$, signs of the Arcturus stream at $V_{\mathrm{az}}=100 \mathrm{~km} \mathrm{~s}^{-1}$, and possibly a further feature at $V_{\mathrm{az}}=75 \mathrm{~km} \mathrm{~s}^{-1}$, which will be investigated more closely in a separate study (Klement et al., in preparation).

However, our main interest here is star streams in the genuine halo star population. Tremaine (1993) has estimated a filling factor by star streams in the halo around the Sun of about 1 , and we expect to find a few genuine halo star streams in the sample. In order to isolate these we have indicated in Fig. 3 the area populated predominantly by stars with disk-like kinematics. The dashed lines indicate the loci of stars with $W= \pm 50 \mathrm{~km} \mathrm{~s}^{-1}$, respectively, and $V_{\mathrm{az}}$ ranging from 20 to $270 \mathrm{~km} \mathrm{~s}^{-1}$, which should bracket the velocity range of thick disk stars. Outside this range we find at velocities $V_{\mathrm{az}}>0$ three distinct isolated features which we propose to interpret as signatures of star streams passing through the solar neighbourhood. Two of them (labelled HB and $S_{1}$ ) are clearly visible on the right side of the bottom right panel of Fig. 2 and in the upper right part of Fig. 3. Their centroid positions in phase space are summarized in Table 1. Interestingly the feature labelled HB is the stream detected by Helmi et al. (1999). Chiba \& Beers (2000) confirmed this discovery later using the same data of Beers et al. (2000) as analyzed in this study, but their "clump stars" were isolated in a different way from our method. Actually we draw confidence in our search technique from the fact that it recovers a previously known star stream in the halo population. The stars in the third feature at $V_{\mathrm{az}}=140 \mathrm{~km} \mathrm{~s}^{-1}$ and $v \approx 30^{\circ}$ have the same orbital parameters (cf. Sect. 3.3) as the stars in the stream newly found by Arifyanto \& Fuchs (2006) and Helmi et al. (2006). The members of this stream have disk-like kinematics, but comparatively large vertical $W$ velocities. Since the inclination angles $v$ depend on the vertical $W$ velocity components, such stars will appear as linearly smeared features in Figs. 3 and 4. The other linear structures in Figs. 3 and 4 are, in our view, also due to this effect. The features in the right lower half in Fig. 3 are therefore very unlikely to be associated with a genuine halo stream.

Stars at negative azimuthal velocities are on retrograde orbits and belong exclusively to the halo population. It is more difficult to discern overdensities in this part of phase space, because the $V$-shaped branches in the $V_{\mathrm{az}}-V_{\Delta \mathrm{E}}$ diagrams in Fig. 2 become very narrow and the inclinations of the orbital planes are evenly distributed over $v$ in Fig. 3. However, we find two further isolated 


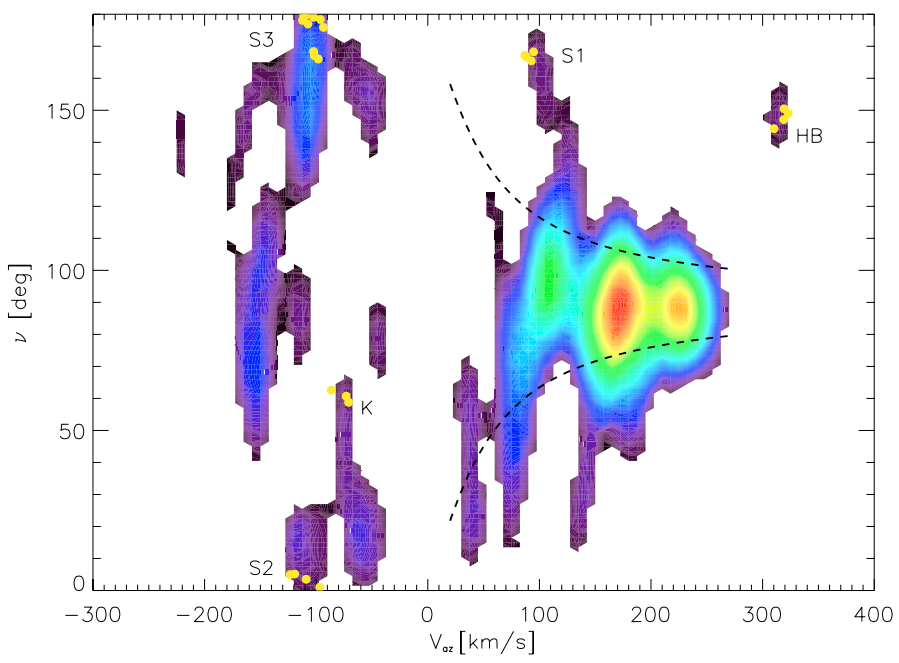

Fig. 3. Projection of the wavelet transform of the phase space distribution of halo stars onto a plane spanned by the inclination angles $v$ of the orbital planes of the stars and their azimuthal velocities $V_{\mathrm{az}}$. A similar colour table as in Fig. 2 has been used, but its peak value at red refers to a density which is a factor of about 3 lower than in Fig. 2. The dashed lines indicate the loci of stars with $W= \pm 50 \mathrm{~km} \mathrm{~s}^{-1}$ and $V_{\mathrm{az}}$ ranging from 20 to $270 \mathrm{~km} \mathrm{~s}^{-1}$ as expected for thick disk stars. The locations of stars assigned to halo overdensities are marked by yellow symbols.

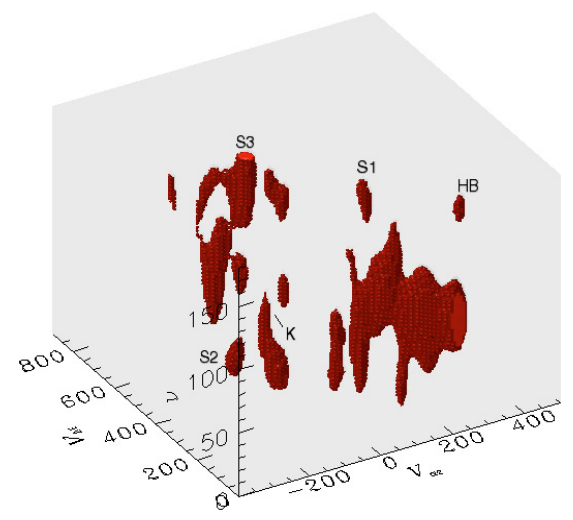

Fig. 4. Three-dimensional rendering of the wavelet transform of the phase space distribution of nearby halo stars. The rendered surface corresponds to the outer countours in Fig. 2.

features in this part of phase space. One is located in the upper left frame of Fig. 2 at negative $V_{\text {az }}$ distinctively above the narrow $V_{\mathrm{az}}-V_{\Delta \mathrm{E}}$ line of the bulk of the stars. This feature is also seen very clearly in the left side of Fig. 3 as a strong overdensity at $v=6^{\circ}$. A second feature can be identified on the left side in the bottom right panel of Fig. 2. Also this feature is seen very clearly as an overdensity on the left side of Fig. 3 at $v=170^{\circ}$. The three proposed streams (labelled $S_{1}, S_{2}$, and $S_{3}$ ) are described here for the first time, to the best of our knowledge. Moreover, we have found signs of Kapteyn's moving group as defined by Eggen (1996), although it is not very prominent. Its position in phase space is given together with that of the other streams in Table 1. Note however that Kapteyn's star itself is not a member of our sample.

\subsection{Monte Carlo simulations}

In Table 1 we give the numbers of stars $N$ which we assign to each overdensity in phase space by our procedure. These are overplotted as yellow symbols in Fig. 3. The numbers of stars in
Table 1. Parameters of the proposed halo star streams.

\begin{tabular}{crcrrrrc}
\hline \hline & $\begin{array}{r}V_{\mathrm{az}} \\
\mathrm{km} \mathrm{s}^{-1}\end{array}$ & $\begin{array}{r}V_{\Delta \mathrm{E}} \\
\mathrm{km} \mathrm{s}^{-1}\end{array}$ & $\begin{array}{r}v \\
\mathrm{deg}\end{array}$ & $N$ & $\begin{array}{r}r_{0} \\
\mathrm{kpc}\end{array}$ & $\begin{array}{r}r_{\text {peri }} \\
\mathrm{kpc}\end{array}$ & $\begin{array}{r}r_{\mathrm{apo}} \\
\mathrm{kpc}\end{array}$ \\
\hline $\mathrm{HB}$ & 320 & 155 & 150 & 4 & 11 & 7.8 & 20 \\
$\mathrm{~S}_{1}$ & 100 & 250 & 165 & 4 & 3.5 & 1.9 & 12 \\
$\mathrm{~S}_{2}$ & -100 & 520 & 6 & 4 & 3.5 & 1.7 & 17 \\
$\mathrm{~S}_{3}$ & -100 & 470 & 170 & 10 & 3.7 & 2.1 & 9.1 \\
$\mathrm{~K}$ & -75 & 440 & 50 & 3 & 2.8 & 1.5 & 10 \\
\hline
\end{tabular}

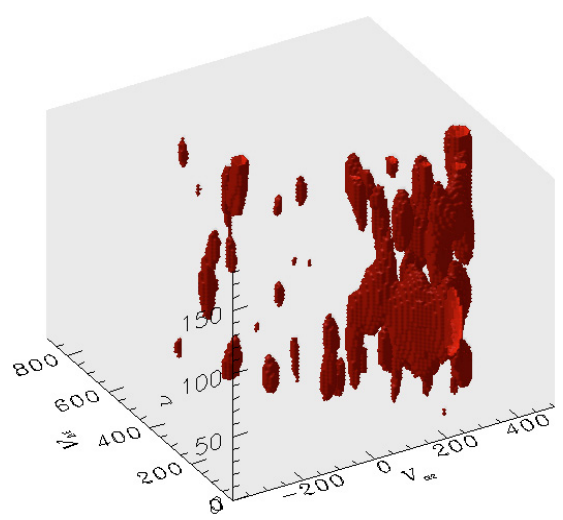

Fig. 5. Three-dimensional rendering of the signal - to noise ratio of the wavelet transform of the phase space distribution of the halo stars at a level of $S / N=2.8$, which corresponds to a confidence level of $99.5 \%$. The expected Poisson noise has been determined with Monte Carlo simulations of a smooth Schwarzschild distribution (cf. Sect. 3.2).

each phase space overdensity are quite small. In order to assess the reality of the overdensities we have performed Monte-Carlo simulations of a smooth velocity distribution of halo stars. We adopted a triaxial Schwarzschild distribution with the velocity dispersions determined by Arifyanto \& Fuchs (2005). The size of the simulated sample was set equal to the observed number of stars with non-disk kinematics. In a typical Monte-Carlo simulation the phase space distribution is rather smooth, but does show overdensities due to Poisson noise. We have run in total 100 Monte Carlo simulations and have calculated the residuals of the wavelet transforms of each individual simulation against the very smooth superposition of all simulations. For each cell in the $V_{\mathrm{az}}-V_{\Delta \mathrm{E}}-v$ cube we have determined the mean and variance of the residuals. Finally we have divided each value of the wavelet transform of the observed phase space distribution of halo stars shown in Figs. 2-4 by the square root of the variance of the numerically simulated Schwarzschild distribution. This procedure gives then the $S / N$ ratio of the detected overdensities. In Fig. 5 a three-dimensional rendering of this ratio at a level of $S / N=2.8$ is shown. At azimuthal velocities less than $V_{\mathrm{az}} \leq-220 \mathrm{~km} \mathrm{~s}^{-1}$ the variances of the residuals are nominally so small, that the $\mathrm{S} / \mathrm{N}$ ratio becomes artificially high. Since Fig. 1 shows absolutely no clustering in this region of phase space, we have masked out this part in Fig. 5. Four of the star streams proposed here are clearly discernible, and are detected at a confidence level of $99.5 \%$ or better. Kapteyn's moving group does not show up at this significance level.

\subsection{Orbital parameters of the halo streams}

We have determined the orbital parameters of the halo streams proposed here and list them in Table 1. Since some of the orbits are fairly eccentric, Dekker's (1976) approximation of Galactic orbits is not accurate enough to give precise estimates of the 
parameters. This does not, however, diminish its power to project even such orbits close together into the same region of phase space, if the orbits are similar to each other! The mean guiding center radii and inner and outer turning radii given in Table 1 have been calculated assuming a logarithmic Galactic gravitational potential $\Phi(r)=V_{\mathrm{LSR}}^{2} \ln (r / 1 \mathrm{kpc})$ which corresponds to a flat rotation curve. The mean guiding center radius of a stream $r_{0}$ is determined by the minimum of the effective potential $\Phi_{\mathrm{eff}}(r)=\Phi(r)+0.5 \cdot L^{2} / r^{2}$ and the turning radii are given by the condition $E=\Phi_{\text {eff }}\left(r_{\text {peri }}, r_{\text {apo }}\right)$, where $E$ denotes the orbital energy. As can be seen from Table 1 stream HB is at present close to its perigalacticon, whereas the other streams dive deep into the inner Galaxy. It would be intriguing if distant counterparts of the locally detected streams could be identified. For instance, Martínez-Delgado et al. (2007) have presented a numerical simulation of the Sagittarius stream which suggests that its leading arm passes in the vicinity of the Sun out of the direction of the North Galactic Pole nearly perpendicular through the Galactic plane, although this was questioned by Newberg et al. (2007). The stream labelled $S_{3}$, which we propose here, has precisely these kinematics. However, its orbital parameters deduced here are difficult to reconcile with the simulated morphology of the Sagittarius stream. Obviously this depends to a large degree on the details of the model adopted for the Galactic potential. Observations as presented here might be helpful to constrain these.

Acknowledgements. We are grateful to the anonymous referee for his helpful comments. B.F. and C.F. thank the Mt. Stromlo observatory for its hospitality, where this research was begun. C.F. acknowledges financial support by the Academy of Finland.

\section{References}

Arifyanto, M. I., \& Fuchs, B. 2005, A\&A, 433, 911 Arifyanto, M. I., \& Fuchs, B. 2006, A\&A, 499, 533

Beers, T. C., Chiba, M., Yoshii, Y., et al. 2000, AJ, 119, 2843

Belokurov, V., Zucker, D. B., Evans, N. W., et al. 2006, ApJ, 642, L137

Chiba, M., \& Beers, T. C. 2000, AJ, 119, 2843

Dehnen, W. 1998, AJ, 115, 2384

Dehnen, W. 2000, AJ, 119, 800

Dehnen, W., \& Binney, J. J. 1998, MNRAS, 298, 387

Dekker, E. 1976, Phys. Rep., 24, 315

Eggen, O. J. 1996, AJ, 112, 1595

Helmi, A. 2004, ApJ, 601, L97

Helmi, A., White, S. D. M., de Zeeuw, P. T., \& Zhao, H. S. 1999, Nature, 402, 53

Helmi, A., Navarro, J. F., Nordström, B., et al. 2006, MNRAS, 365, 1309

Ibata, R. A., Irwin, M. J., Lewis, G. F., \& Stolte, A. 2001, ApJ, 547, L133

Ibata, R. A., Irwin, M. J., Lewis, G. F., Ferguson, A., \& Tanvir, N. 2003, MNRAS, 340, L21

Law, D. R., Johnston, K. V., \& Majewski, S. R. 2005, ApJ, 619, 807

Majewski, S. R., Skrutskie, M., Weinberg, M., \& Ostheimer, J. 2003, ApJ, 599, 1082

Martínez-Delgado, D., Aparicio, A., Gómez-Flechoso, M. A., \& Carrera, R. 2001, ApJ, 601, 242

Martínez-Delgado, D., Peñarrubia, J., Jurić, M., Alfaro, E. J., \& Ivezić, Z. 2007, ApJ, 660, 1264

Navavarro, J. F., Helmi, A., \& Freeman, K. C. 2004, ApJ, 601, 43

Newberg, H. J., Yanny, B., Cole, N., et al. 2007 [arXiv: 0706. 339]

Peñarrubia, J., Martínez-Delgado, D., \& Rix, H.-W. 2005, A\&A, 626, 128

Quillen, A., \& Minchev, I. 2005, AJ, 130, 576

Skuljan, J., Hearnshaw, J. B., \& Cottrell, P. L. 1999, MNRAS, 308, 731

Tremaine, S. 1993, AIP Conf. Proc., 278, 599

Yanny, B., Newberg, H. J., Grebel, E. K., et al. 2003, ApJ, 588, 824 